\title{
Ginsenoside Rc protects against UVB-induced photooxidative damage in epidermal keratinocytes
}

\author{
YURI OH ${ }^{1}$, HYE-WON LIM ${ }^{2}$, KWANG HARK PARK ${ }^{1}$, YU-HUA HUANG ${ }^{2}$, \\ JI-YOUNG YOON ${ }^{3}$, KYUNGHOON KIM ${ }^{1}$ and CHANG-JIN LIM ${ }^{2,4}$
}

\author{
${ }^{1}$ Department of Biological Sciences, College of Natural Sciences, Kangwon National University, Chuncheon, Gangwon 24341; \\ ${ }^{2}$ Shebah Biotech Inc., G-Tech Village, Chuncheon, Gangwon 24398; ${ }^{3}$ Jeonju AgroBio-Materials Institute, \\ Jeonju, Jeollabuk 54810; ${ }^{4}$ Department of Biochemistry, College of Natural Sciences, \\ Kangwon National University, Chuncheon, Gangwon 24341, Republic of Korea
}

Received August 17, 2016; Accepted May 3, 2017

DOI: $10.3892 / \mathrm{mmr} .2017 .6943$

\begin{abstract}
Ginsenoside Rc (Rc) is a major ginsenoside isolated from Panax ginseng, and has exhibited pharmacological effects on skin cells. The present study aimed to investigate the putative skin-protective properties of Rc, including its anti-photoaging and barrier function-protective effects, in human HaCaT keratinocytes exposed to UVB radiation. The protective properties of Rc were evaluated through the assessment of keratinocyte viability, reactive oxygen species (ROS) production, total glutathione (GSH) and superoxide dismutase (SOD) activity, caspase-14, matrix metalloproteinase (MMP)-2 and -9 activity, and MMP-2, MMP-9 and filament aggregating protein (filaggrin) expression following UVB irradiation. Treatment with Rc was revealed to prevent the UVB-induced increase in ROS production and pro-MMP-2 and -9 levels in HaCaT keratinocytes. In addition, treatment with Rc resulted in enriched GSH contents and enhanced SOD activity following exposure to UVB radiation. Furthermore, Rc treatment enhanced caspase-14 activity and counteracted the UVB-induced downregulation in filaggrin expression. However, no significant difference was identified between Rc-treated and normal groups in terms of keratinocyte viability, regardless of exposure to radiation. The present findings suggested that Rc may exert anti-photoaging and barrier function-protective effects in keratinocytes, and
\end{abstract}

Correspondence to: Professor Kyunghoon Kim, Department of Biological Sciences, College of Natural Sciences, Kangwon National University, 192-1 Hyoja-2-dong, Chuncheon, Gangwon 24341, Republic of Korea

E-mail:kkim@kangwon.ac.kr

Professor Chang-Jin Lim, Department of Biochemistry, College of Natural Sciences, Kangwon National University, 192-1 Hyoja-2-dong, Chuncheon, Gangwon 24341, Republic of Korea

E-mail: cjlim@kangwon.ac.kr

Key words: caspase-14, filaggrin, ginsenoside Rc, glutathione, reactive oxygen species, skin barrier function, superoxide dismutase, $\mathrm{UVB}$ radiation thus protect the skin against photooxidative stress induced by exposure to UV radiation.

\section{Introduction}

Ginsenosides are a class of natural products isolated from Panax ginseng and are responsible for the majority of its pharmacological properties. Ginsenosides are classified into 4 categories with regards to the chemical structure of their aglycones: i) Protopanaxadiol (PPD)-; ii) protopanaxatriol (PPT)-; iii) oleanolic acid; and iv) ocotillol-type ginsenosides (1). Ginsenoside Rc (Rc; Fig. 1) is a PPD-type bioactive ginsenoside, which are present at high concentrations in several commercially available ginseng products (2).

$\mathrm{Rc}$ has been reported to interfere with the intracellular production of reactive oxygen species (ROS); however, its actions remain controversial and they may vary depending on cell type $(3,4)$. Under conditions of tert-butylhydroperoxide-induced oxidative stress in HEK293 cells, Rc was reported to attenuate ROS generation, through the upregulation of catalase expression, a forkhead box protein $\mathrm{O} 1$ (FOXO1)-targeting gene (3). In addition, Rc was demonstrated to directly scavenge superoxide free radicals in HEK293 cells (3). Antidiabetic properties have also been reported for Rc, as it was revealed to potentiate glucose uptake via increasing ROS production and activating 5' adenosine monophosphate-activated protein kinase and p38 mitogen-activated protein kinase, in an insulin-independent pathway (4). Therefore, the present study aimed to evaluate the putative antioxidative properties of Rc in skin cells.

UVB radiation has been identified as a major cause of photoaging, as it initiates photooxidative reactions that disrupt the redox balance of skin cells and increase intracellular ROS levels, thus leading to oxidative stress (5). Matrix metalloproteinases (MMPs) are responsible for the degradation of extracellular matrix (ECM) components that constitute the dermal connective tissue (6). A UVB-induced increase in ROS production may stimulate MMPs through redox-regulated transcription factors (7). Photoaging is the result of the degradation of dermal ECM due to enhanced MMP activation and suppressed collagen biosynthesis under conditions of oxidative 
stress (8). The UV-induced gelatinolytic activity of MMP-2 and MMP-9 is involved in the development of UV-induced skin damage, including skin thickening and wrinkle formation (9). UVB irradiation has previously been demonstrated to increase the expression of MMP-2 and MMP-9 and potentiate their gelatinolytic activity in human HaCaT keratinocytes (8).

Filament aggregating protein (filaggrin) is an important component of the cornified cell envelope (CE) of the epidermal stratum corneum ( $S C$ ), and is initially synthesized as profilaggrin, a $500-\mathrm{kDa}$ highly phosphorylated His-rich protein containing 10-12 tandemly arranged filaggrin repeats in its central region (10). Monomeric filaggrins are generated by proteolytic cleavage and dephosphorylation and trigger the aggregation of keratin filaments. Filaggrin has an important function during water retention in SC, whereas its mutations have been associated with natural moisturizing factor (NMF) deficiency in the SC, leading to skin barrier dysfunction (11). A previous study has also reported that silencing of filaggrin expression may impair the skin barrier functions of normal human epidermal keratinocytes, primarily via targeting the $\mathrm{CE}$ and triggering immune responses (12). The downregulation or complete loss of filaggrin expression has been previously reported to disturb skin barrier function and enhance the percutaneous transfer of allergens, thus suggesting that filaggrin may have a protective function against the entry of foreign environmental substances (13).

Caspase-14 is a Cys-specific proteinase localized in stratified epithelia, including the skin, and is involved in the production of filaggrin monomers and the synthesis of NMFs in the skin (14). Caspase-14 is activated only in terminally differentiated keratinocytes, and its downregulation has been reported to lead to impairments in skin barrier function (15).

The findings of the present study suggested that Rc may possess protective properties against UVB-induced photooxidative damage and may exert anti-photoaging and barrier function-protective effects in keratinocytes.

\section{Materials and methods}

Chemicals. Rc (purity, $\geq 98 \%$ ) was obtained from Ambo Institute (Daejeon, Korea; www.ambo.co.kr). Bradford reagent, MTT solution, 2',7'-dichlorodihydrofluorescein diacetate (DCFH-DA), dihydrorhodamine 123 (DHR-123), dihydroethidium (DHE), 5,5'-dithiobis (2-nitrobenzoic acid) (DTNB), glutathione reductase (GR) and nicotinamide-adenine dinucleotide phosphate (NADPH) were purchased from Sigma-Aldrich; Merck KGaA (Darmstadt, Germany). Ac-Trp-Glu-His-Asp-7-amino-4-methylcoumarin (Ac-WEHD-MCA) was purchased from Peptide Institute, Inc. (Osaka, Japan). Cell lysis buffer was obtained from Promega Corporation (Madison, WI, USA).

Cell culture. The human HaCaT keratinocyte cell line was purchased from American Type Culture Collection (Manassas, VA, USA). Cells were cultured in Dulbecco's modified Eagle's medium (DMEM; HyClone; GE Healthcare Life Science, Logan, UT, USA) supplemented with 10\% heat-inactivated fetal bovine serum (FBS; HyClone; GE Healthcare Life Sciences), $100 \mathrm{U} / \mathrm{ml}$ penicillin and $100 \mu \mathrm{g} / \mathrm{ml}$ streptomycin, and maintained at $37^{\circ} \mathrm{C}$ in a humidified atmosphere with $5 \% \mathrm{CO}_{2}$.

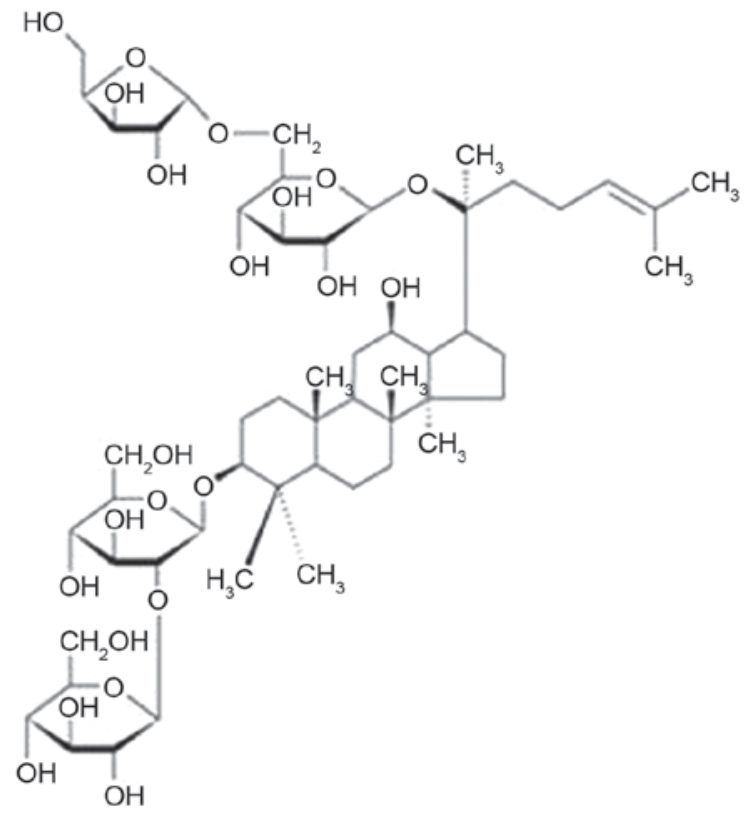

Figure 1. Chemical structure of ginsenoside Rc.

UVB irradiation. A VL-6 M ultraviolet lamp (peak, $312 \mathrm{~nm}$; Vilber Lourmat, Marne-la-Vallée, France) was the source of UVB radiation, and was used with a VLX-3 $\mathrm{W}$ radiometer (Vilber Lourmat) equipped with a CX-312 sensor (bandwidth, 280-320 nm; Vilber Lourmat). Prior to the irradiation, $1 \times 10^{5}$ cells, grown overnight and washed twice with $1 \mathrm{ml}$ PBS, were resuspended in $1 \mathrm{ml}$ FBS-free DMEM. HaCaT keratinocytes at $25^{\circ} \mathrm{C}$ were irradiated with solar simulated UVB radiation at $70 \mathrm{~mJ} / \mathrm{cm}^{2}$ for $2 \mathrm{~min}$, an intensity estimated to induce oxidative stress in preliminary experiments (data not shown).

Preparation of cell lysates. Adherent cells were harvested using a cell scraper, washed twice with PBS and maintained on ice for $5 \mathrm{~min}$. Following centrifugation at $3,000 \mathrm{x} \mathrm{g}$ for $10 \mathrm{~min}$ at room temperature, the cell pellets were dissolved in cell lysis buffer containing $50 \mathrm{mM}$ HEPES (pH 7.5), 10\% sucrose and $0.1 \%$ Triton X-100, and maintained on ice for $30 \mathrm{~min}$. Following centrifugation at $10,000 \mathrm{xg}$ for $15 \mathrm{~min}$ at $4^{\circ} \mathrm{C}$, the supernatants were collected. Protein concentration in cell lysates was determined by the Bradford protein assay as previously described (16), using bovine serum albumin (Sigma-Aldrich; Merck KGaA) as the standard.

Intracellular ROS production. The ROS-sensitive fluorescent probe DCFH-DA produces 2',7'-dichlorofluorescein $\left(\lambda_{\text {excitation }}\right.$, $485 \mathrm{~nm} ; \lambda_{\text {emission }}, 530 \mathrm{~nm}$ ) upon enzymatic reduction and oxidation by ROS (17). DHR-123 and DHE are additional ROS probes which produce rhodamine $123\left(\lambda_{\text {excitation }}, 500 \mathrm{~nm} ; \lambda_{\text {emission}}\right.$, $535 \mathrm{~nm})$ and 2-hydroxyethidium $\left(\lambda_{\text {excitation }}, 480 \mathrm{~nm} ; \lambda_{\text {emission }}\right.$, $525 \mathrm{~nm}$ ), respectively, upon reaction with ROS (18). Prior to the treatment, $1 \times 10^{5}$ cells, grown overnight and washed twice with $1 \mathrm{ml}$ PBS, were resuspended in $1 \mathrm{ml}$ FBS-free DMEM. HaCaT keratinocytes were incubated with $\operatorname{Rc}(0,5,12$ and $30 \mu \mathrm{M})$ and $20 \mu \mathrm{M}$ DCFH-DA, $5 \mu \mathrm{M}$ DHR-123 or $5 \mu \mathrm{M}$ DHE for $30 \mathrm{~min}$ at $37^{\circ} \mathrm{C}$. Then, the cells were washed twice with $1 \mathrm{ml} \mathrm{FBS}$-free DMEM, dissolved in $1 \mathrm{ml} \mathrm{FBS-free} \mathrm{DMEM} \mathrm{and} \mathrm{irradiated} \mathrm{with}$ 
$70 \mathrm{~mJ} / \mathrm{cm}^{2} \mathrm{UVB}$ radiation, if required. The appropriate control cells did not receive Rc and were treated with or without UVB irradiation. Intracellular ROS levels were determined via quantification of the fluorescence of the samples using the Synergy HTX Multi-Mode microplate reader (BioTek Instruments Inc., Winooski, VT, USA).

Cell viability assay. In order to investigate the cytotoxic effects of UVB irradiation on HaCaT keratinocytes, and the putative cytoprotective properties of Rc, cell viability was evaluated using an MTT assay, which reflects cellular metabolic activity (19). A total of $1 \times 10^{5} \mathrm{HaCaT}$ keratinocytes, grown overnight and washed twice with $1 \mathrm{ml}$ PBS, were resuspended in FBS-free DMEM and treated with Rc for $1 \mathrm{~h}$. If necessary, the cells were irradiated following the Rc treatment. The quantity of formazan dissolved in dimethyl sulfoxide, generated from the reduction of MTT by the mitochondria of viable cells, was determined by the absorbance at $540 \mathrm{~nm}$ using the Synergy HTX Multi-Mode microplate reader.

Gelatin zymography. The proteolytic activity of MMP-2 and MMP-9 in conditioned media, obtained through centrifugation at $10,000 \mathrm{x} \mathrm{g}$ for $10 \mathrm{~min}$ at $4^{\circ} \mathrm{C}$, was assessed using gelatin zymographic analysis (20) with a slight modification. Briefly, following staining of the SDS-polyacrylamide gel with $0.1 \%$ Coomassie Brilliant Blue R-250, the areas of gelatinolytic activity were identified as clear white bands against a darkly stained background. Molecular mass markers were used to verify the MMP-2 and MMP-9 activity bands at 72 and $92 \mathrm{kDa}$, respectively. The band strength was determined with densitometry using ImageJ 1.48 software (National Institutes of Health, Bethesda, MD, USA).

Western blot analysis. Western blot analysis was used to assess the protein expression levels of MMP-2, MMP-9 and filaggrin in keratinocyte lysates. The following primary antibodies were used: Anti-MMP-2 (cat. no. ALX-210-753; Enzo Life Sciences Inc., Farmingdale, NY, USA), anti-MMP-9 (cat. no. 3852S; Cell Signaling Technology Inc., Danvers, MA, USA), anti-filaggrin (cat. no. SC-30229; Santa Cruz Biotechnology Inc., Dallas, TX, USA) and anti-GAPDH (cat. no. LF-PA0212; Young In Frontier Co., Ltd., Seoul, Korea). Cellular lysates (protein content, $10 \mu \mathrm{g} /$ lane) were separated using SDS-PAGE on a $10 \%$ (w/v) gel and electrotransferred to a polyvinylidene fluoride membrane (Sigma-Aldrich; Merck KGaA). The membranes, after blocking with 2\% BSA (Sigma-Aldrich; Merck KGaA) for $1 \mathrm{~h}$ at room temperature, were probed with the primary antibodies at a 1:1,000 dilution at $4^{\circ} \mathrm{C}$ overnight, and subsequently incubated with horseradish peroxidase-conjugated secondary antibodies (goat anti-rabbit immunoglobulin G; 1:1,000; cat. no. ADI-SAB-300; Enzo Life Sciences Inc.) at room temperature for $1 \mathrm{~h}$. Protein bands were visualized by enhanced chemiluminescence using the WESTSAVE Femto detection kit (cat. no. LF-QC0109; Young In Frontier Co., Ltd.). GAPDH was used as the loading control. Densities of the protein bands were determined using ImageJ software.

Total glutathione (GSH) contents. Total GSH contents in keratinocyte lysates were evaluated using a GR-coupled enzymatic recycling assay, as previously described (21). Lysates were incubated at $25^{\circ} \mathrm{C}$ for $5 \mathrm{~min}$ in $200 \mu \mathrm{l}$ reaction mixture, which contained $175 \mathrm{mM} \mathrm{KH_{2 }} \mathrm{PO}_{4}, 6.3 \mathrm{mM}$ EDTA, $0.21 \mathrm{mM}$ NADPH, $0.6 \mathrm{mM}$ DTNB, and $0.5 \mathrm{U} / \mathrm{ml} \mathrm{GR}$. The absorbance of the samples at $412 \mathrm{~nm}$ was determined using a microplate reader. Total GSH contents, expressed as $\mu \mathrm{g} / \mathrm{mg}$ protein, were determined using a GSH standard curve and normalized to the total protein content of cell lysates.

Superoxide dismutase (SOD) activity assay. Total SOD activity in keratinocyte lysates was determined based upon the reduction of cytochrome $c$ with a xanthine/xanthine oxidase system, as previously described (22). The reaction mixture (200 $\mu \mathrm{l})$ contained $50 \mathrm{mM}$ PB (pH 7.4), $0.01 \mathrm{U} / \mathrm{ml}$ xanthine oxidase, $0.1 \mathrm{mM}$ EDTA, $1 \mu \mathrm{M}$ catalase, $0.05 \mathrm{mM}$ xanthine, $20 \mu \mathrm{M}$ cytochrome $c$ and the cell lysate. The mixture was incubated at $25^{\circ} \mathrm{C}$ for $10 \mathrm{~min}$. The absorbance of each sample was measured at $550 \mathrm{~nm}$ using a microplate reader. SOD activity was normalized to total protein contents of the lysates, and expressed as $\Delta \mathrm{A}_{550} / \mathrm{min} / \mathrm{mg}$ protein.

Caspase-14 activity assay. Caspase-14 activity in keratinocyte lysates was assessed using Ac-WEHD-MCA (Peptide Institute Inc.) as a fluorogenic substrate, as previously described (15). The reaction mixture $(95 \mu \mathrm{l})$ contained $0.1 \mathrm{M}$ HEPES buffer (pH 7.5), $0.06 \mathrm{M} \mathrm{NaCl}, 0.01 \%$ CHAPS detergent, $5 \mathrm{mM}$ dithiothreitol, 1.3 M sodium citrate, and $10 \mu \mathrm{M}$ Ac-WEHD-MCA. Cell lysate $(5 \mu \mathrm{l})$ was added to the mixture and incubated at room temperature for $30 \mathrm{~min}$. The intensity of fluorescence ( $\lambda_{\text {excitation }}, 355 \mathrm{~nm} ; \lambda_{\text {emission }}, 460 \mathrm{~nm}$ ) was measured using the Synergy HTX Multi-Mode microplate reader. Caspase-14 activity was normalized to the protein contents of the lysates.

Statistical analysis. Data are expressed as the mean \pm standard deviation. The statistical significance of the differences between groups was assessed using one-way analysis of variance followed by a post hoc Tukey's honest significant difference test for multiple comparisons. SPSS version 16.0 for Windows was used to perform the statistical analyses (SPSS, Inc., Chicago, IL, USA). $\mathrm{P}<0.05$ was considered to indicate a statistically significant difference.

\section{Results}

Rc treatment suppresses UVB-induced ROS production. $\mathrm{HaCaT}$ keratinocytes were treated with various concentrations $(0,5,12$ or $30 \mu \mathrm{M})$ of Rc prior to UVB irradiation and intracellular ROS generation was assessed using ROS-sensitive probes. The DCFH-DA assay demonstrated that UVB irradiation led to a $~ 8.4$-fold elevation in intracellular ROS levels compared with non-irradiated control cells (Fig. 2A). Rc was revealed to prevent UVB-induced increase in ROS production, as ROS levels were reduced to $65.7,45.6$ and $34.6 \%$ of UVB-irradiated cells following treatment with 5, 12 and $30 \mu \mathrm{M}$ Rc, respectively (Fig. 2A). The DHR-123 assay indicated that UVB induced a $\sim 6.0$-fold increase in ROS production compared with non-irradiated control cells, whereas Rc attenuated the UVB-induced elevation in ROS levels in a dose-dependent manner (Fig. 2B). Furthermore, DHE was also used as a ROS probe, and similar results were obtained, as Rc was revealed to 
A

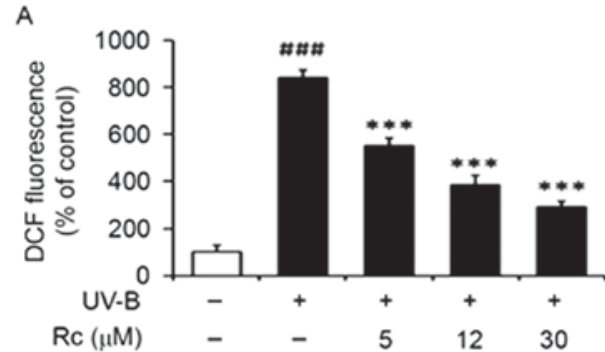

B

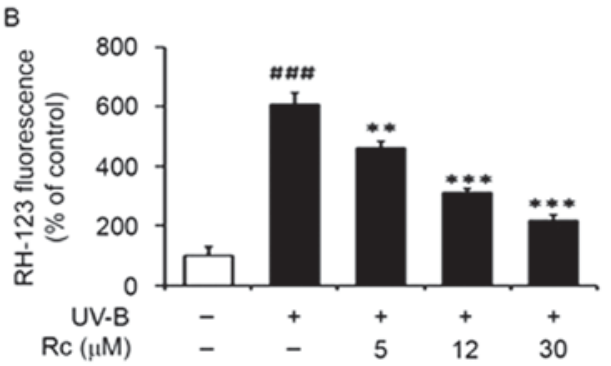

C

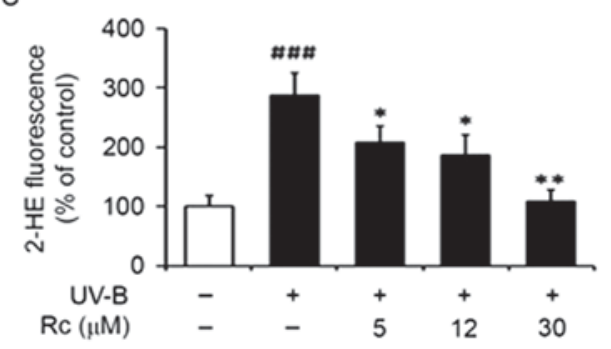

Figure 2. Rc treatment suppresses UVB-induced ROS generation in human HaCaT keratinocytes. HaCaT cells were pretreated with 0, 5, 12 and $30 \mu \mathrm{M}$ Rc for $30 \mathrm{~min}$ prior to UVB irradiation. ROS levels were quantified using (A) DCFH-DA, (B) DHR-123 and (C) DHE and expressed as relative (A) DCF, (B) RH-123 and (C) 2-HE fluorescence. Data are expressed as the mean \pm standard deviation of three independent experiments.

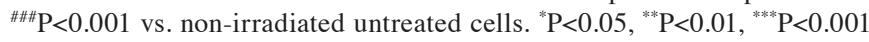
vs. untreated irradiated cells. Rc, ginsenoside Rc; ROS, reactive oxygen species; DCFH-DA, 2',7'-dichlorodihydrofluorescein diacetate; DHR-123, dihydrorhodamine 123; DHE, dihydroethidium; DCF, 2',7'-dichlorofluorescein; RH-123, rhodamine 123; 2-HE, 2-hydroxyethidium.

suppress UVB-induced ROS production (Fig. 2C). The present findings suggested that Rc may suppress intracellular ROS generation following exposure to UVB radiation in human keratinocytes.

$R c$ treatment does not exert cytotoxic effects in keratinocytes. An MTT assay was used to investigate the effects of UVB exposure and treatment with Rc on the viability of $\mathrm{HaCaT}$ keratinocytes. The present findings demonstrated that $\mathrm{Rc}$ did not exert cytotoxic actions on $\mathrm{HaCaT}$ cells in the concentration range that was used (Fig. 3A). In addition, UVB irradiation did not affect the viability of keratinocytes (Fig. 3B), and Rc did not appear to influence the viability of UVB-irradiated cells (Fig. 3B). The present findings suggested that UVB radiation, at the conditions used in the present study, and Rc do not have a toxic effect on HaCaT keratinocytes.

Rc treatment suppresses $M M P-2$ and $M M P-9$ activity following UVB irradiation. In accordance with a previous study (8), UVB irradiation potentiated the proteolytic activity
A

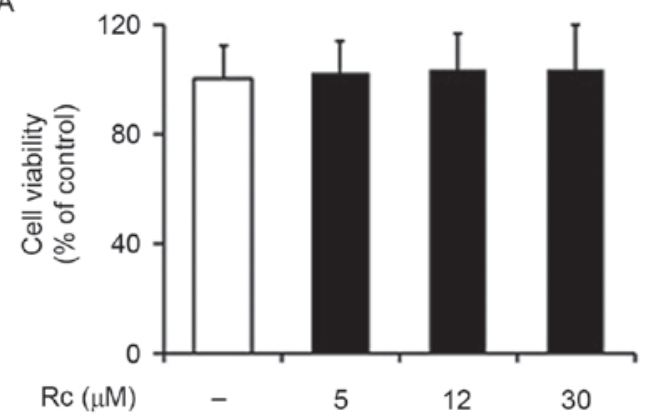

B

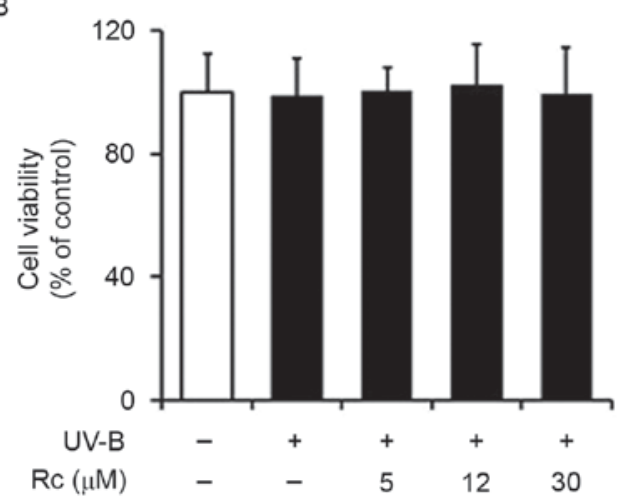

Figure 3. Rc treatment does not affect the viability of human HaCaT keratinocytes. (A) HaCaT cells were treated with $0,5,12$ and $30 \mu \mathrm{M}$ Rc for $30 \mathrm{~min}$. (B) $\mathrm{HaCaT}$ cells were pretreated with $0,5,12$ and $30 \mu \mathrm{M} \mathrm{Rc}$ for $30 \mathrm{~min}$ prior to UVB irradiation. MTT assay was used to evaluate cellular viability and results are expressed as \% of control. Data are expressed as the mean \pm standard deviation. Rc, ginsenoside Rc.

of MMP-2 in conditioned media (Fig. 4A). Notably, treatment of keratinocytes with Rc attenuated the UVB-induced increase in MMP-2 activity, as 5, 12 and $30 \mu \mathrm{M} \mathrm{Rc}$ were revealed to reduce MMP-2 activity to $39.3,21.0$ and $8.0 \%$ of that in untreated irradiated cells, respectively (Fig. 4A). Similarly, keratinocytes exposed to UVB radiation exhibited significantly increased MMP-9 gelatinolytic activity (Fig. 4B), whereas Rc counteracted this effect, as 5, 12 and $30 \mu \mathrm{M} \mathrm{Rc}$ were demonstrated to suppress MMP-9 activity to 27.8, 21.1 and $15.4 \%$ compared with untreated irradiated cells, respectively. The present findings suggested that treatment of keratinocytes with Rc may counteract the increase in proteolytic activity following UVB exposure in vitro.

Rc treatment prevents the UVB-induced upregulation of $M M P-2$ and MMP-9 protein expression. Western blot analysis demonstrated that following exposure to UVB radiation, HaCaT keratinocytes exhibited significantly increased MMP-2 protein expression levels (3.7-fold in non-irradiated control cells; Fig. 5A). Notably, treatment with 5, 12 and $30 \mu \mathrm{M} \mathrm{Rc}$ reduced MMP-2 protein expression levels in UVB-treated cells to $57.2,40.1$ and $11.7 \%$ of those in untreated irradiated cells, respectively (Fig. 5A). Similarly, UVB irradiation led to a significant potentiation of MMP-9 protein expression, whereas Rc was demonstrated to attenuate the UVB-induced MMP-9 upregulation in a dose-dependent manner (Fig. 5B). The present findings suggested that treatment with Rc may reduce the UVB-induced upregulation of MMP-2 and MMP-9 protein expression in human keratinocytes. 
A

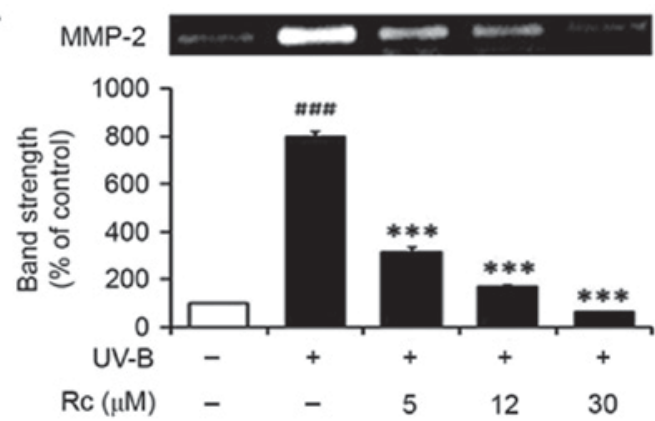

B

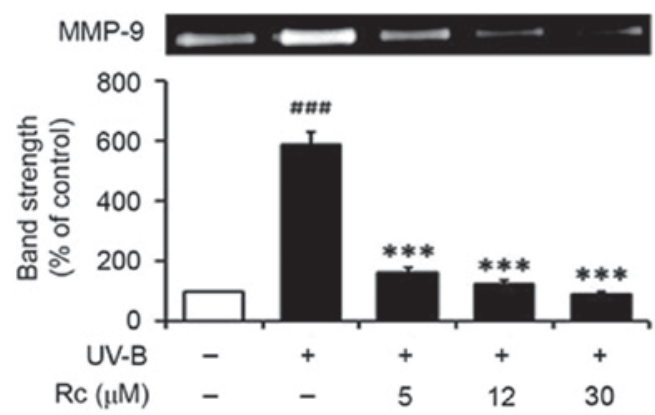

C

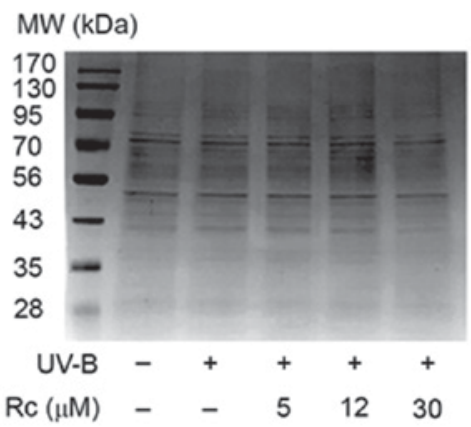

Figure 4. Rc treatment suppresses MMP-2 and MMP-9 activity following UVB irradiation. Human HaCaT keratinocytes were pretreated with 0, 5, 12 and $30 \mu \mathrm{M}$ Rc for 30 min prior to UVB irradiation. The proteolytic activity of (A) MMP-2 and (B) MMP-9 was assessed in conditioned media using gelatin zymography and is expressed as \% of control. (C) Equal loading of conditioned media was confirmed following staining with coomassie blue. Data are expressed as the mean \pm standard deviation of at least three independent experiments. ${ }^{\# \#} \mathrm{P}<0.001$ vs. non-irradiated untreated cells. ${ }^{* * *} \mathrm{P}<0.001$ vs. untreated irradiated cells. Rc, ginsenoside Rc; MMP, matrix metalloproteinase; MW, molecular weight.

A

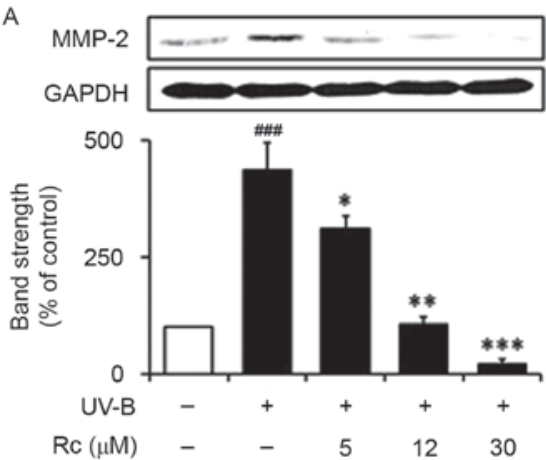

B
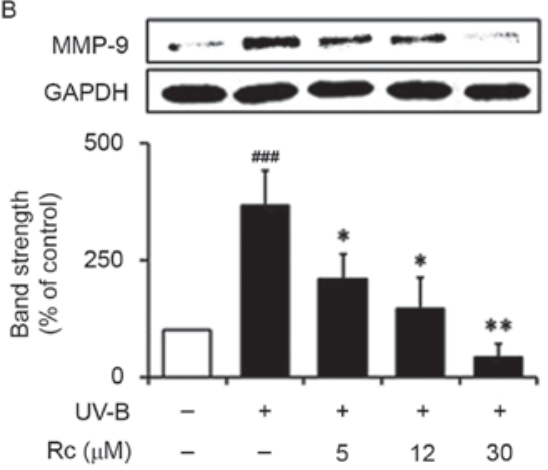

Figure 5. Rc treatment prevents the UVB-induced upregulation of MMP-2 and MMP-9 protein expression levels. Human HaCaT keratinocytes were pretreated with $0,5,12$ and $30 \mu \mathrm{M}$ Rc for 30 min prior to UVB irradiation. Western blot analysis was used to assess the protein expression levels of (A) MMP-2 and (B) MMP-9 in cell lysates. Blots were semi-quantified using densitometry and relative band intensity is expressed as \% of control. Representative blots are included. Data are expressed as the mean \pm standard deviation of three independent experiments. ${ }^{\# \#} \mathrm{P}<0.001$ vs. non-irradiated untreated cells. ${ }^{*} \mathrm{P}<0.05,{ }^{* *} \mathrm{P}<0.01,{ }^{* * *} \mathrm{P}<0.001$ vs. untreated irradiated cells. Rc, ginsenoside Rc; MMP, matrix metalloproteinase.
Rc treatment prevents UVB-induced GSH depletion. The present study demonstrated that total GSH contents were significantly reduced in UVB-irradiated $\mathrm{HaCaT}$ keratinocytes (Fig. 6A), which was in accordance with a previous study (23). Notably, following treatment with 5,12 and $30 \mu \mathrm{M} \mathrm{Rc}$, total GSH levels were increased by 1.9-, 2.0- and 2.3-fold, respectively, compared with untreated irradiated cells (Fig. 6A). These findings suggested that the increased GSH contents may be implicated in the molecular mechanisms underlying the effects of Rc against UVB-induced keratinocyte damage.

Rc treatment prevents the UVB-induced suppression of SOD activity. UVB irradiation was revealed to significantly suppress total SOD activity in HaCaT keratinocytes (Fig. 6B). However, following treatment with 5,12 and $30 \mu \mathrm{M} \mathrm{Rc}$, total SOD activity was potentiated by 2.0-,2.9- and 3.7-fold, respectively, compared with untreated irradiated cells (Fig. 6B). The present findings suggested that Rc may counteract the UVB-induced impairments in SOD activity in keratinocytes in vitro.

Rc treatment enhances caspase-14 activity and upregulates filaggrin protein expression. Following exposure to UVB radiation, the activity of caspase-14 was significantly reduced (75.0\% of that in non-irradiated control cells; Fig. 7A). Notably, treatment with 5, 12 and $30 \mu \mathrm{M}$ Rc was demonstrated to potentiate the activity of caspase-14 by 2.2- . 2.5- and 2.8-fold, respectively, compared with untreated irradiated keratinocytes (Fig. 7A).

The protein expression levels of filaggrin were revealed to be significantly reduced in UVB-irradiated $\mathrm{HaCaT}$ cells compared with non-irradiated control cells $(66.7 \%$ of those in non-irradiated cells; Fig. 7B). Following treatment with 5, 12 

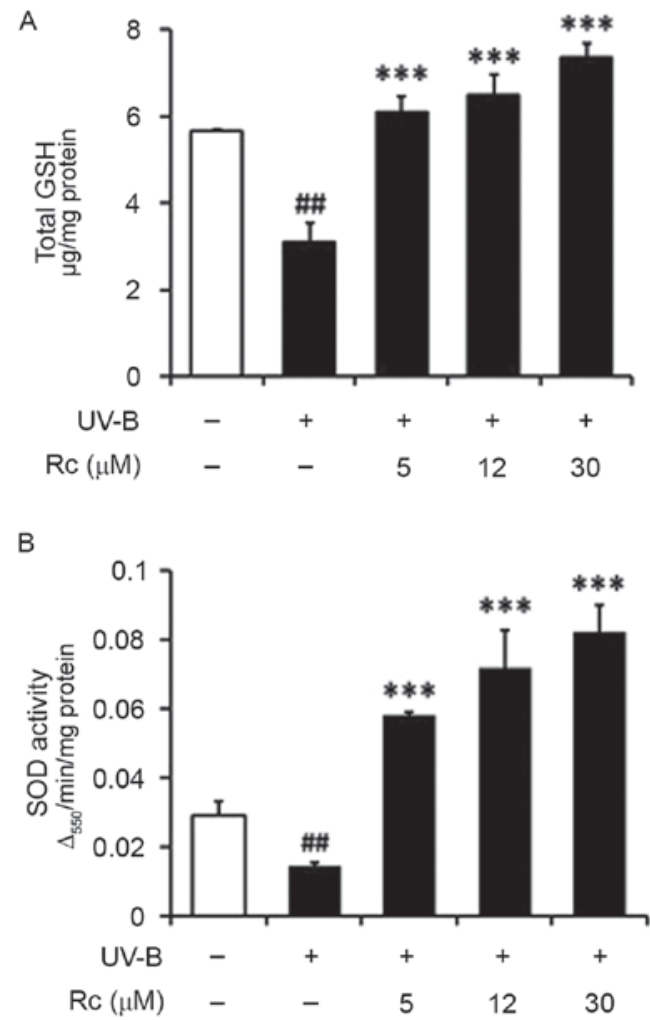

Figure 6. Rc prevents UVB-induced impairments in the endogenous antioxidant mechanisms. Human $\mathrm{HaCaT}$ keratinocytes were pretreated with 0,5 , 12 and $30 \mu \mathrm{M} \mathrm{Rc}$ for $30 \mathrm{~min}$ prior to UVB irradiation. (A) Rc prevented the UVB-induced GSH depletion. (B) Rc prevented the UVB-induced suppression of SOD activity. Data are expressed as the mean \pm standard deviation. ${ }^{\# \#} \mathrm{P}<0.01$ vs. non-irradiated untreated cells. ${ }^{* * *} \mathrm{P}<0.001$ vs. untreated irradiated cells. Rc, ginsenoside Rc; GSH, glutathione; SOD, superoxide dismutase.

and $30 \mu \mathrm{M} \mathrm{Rc}$, filaggrin protein expression was significantly upregulated by 4.5-, 5.4- and 7.8-fold, respectively, compared with untreated irradiated keratinocytes (Fig. 7B). The present findings suggested that treatment with Rc may counteract UVB-induced impairments in skin barrier function under conditions of photooxidative stress.

\section{Discussion}

The present study aimed to evaluate the putative antioxidative properties of Rc in UVB-exposed human keratinocytes in vitro. DCFH-DA, DHR-123 and DHE are fluorescent ROS probes and were used to investigate the effects of Rc treatment on UVB-induced ROS production. DCFH-DA, originally used as a specific probe for hydrogen peroxide, also reacts with other ROS, including hydroxyl and peroxy radicals (18). DHR-123 reacts with hydrogen peroxide in the presence of peroxidases and may be oxidized by other oxidants, including peroxynitrite anions and hypochlorous acid (18). DHE is used as a probe for the detection of superoxide radicals, whereas it is also oxidized by hydrogen peroxide via non-specific peroxidase catalysis (18). The present study demonstrated that UVB irradiation led to increased ROS levels in HaCaT keratinocytes. These findings are in accordance with a previous study (24), which reported that UVB radiation induced the production of superoxide radicals in epidermal keratinocytes, which are subsequently converted to other ROS species, including

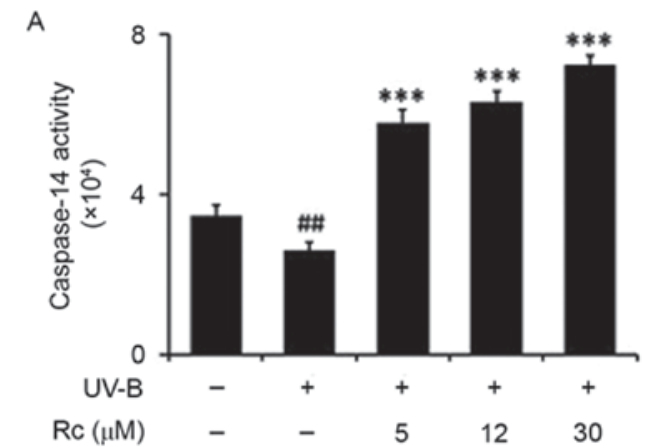

B

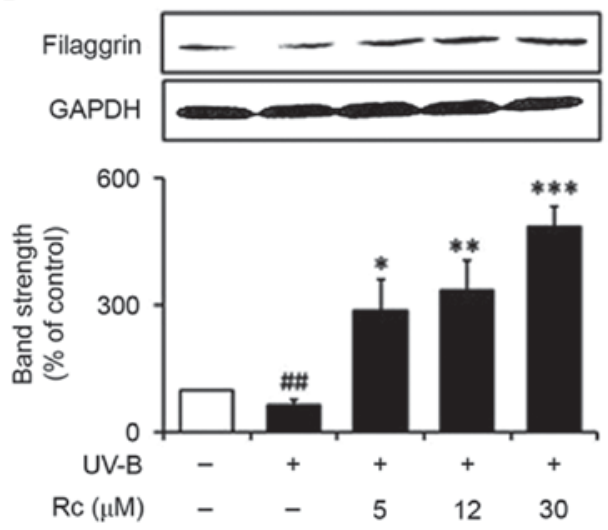

Figure 7. Rc enhances caspase-14 activity and upregulates filaggrin protein expression. Human $\mathrm{HaCaT}$ keratinocytes were pretreated with 0, 5, 12 and $30 \mu \mathrm{M}$ Rc for $30 \mathrm{~min}$ prior to UVB irradiation. (A) Caspase-14 activity was determined using a fluorogenic assay and is expressed as an increase in fluorescence. (B) Western blot analysis was used to assess the protein expression levels of filaggrin. Blots were semi-quantified using densitometry and relative band intensity is expressed as \% of control. Data are expressed as the mean \pm standard deviation. ${ }^{\# \#} \mathrm{P}<0.01$ vs. non-irradiated untreated cells. ${ }^{*} \mathrm{P}<0.05,{ }^{* *} \mathrm{P}<0.01,{ }^{* * *} \mathrm{P}<0.001$ vs. untreated irradiated cells. Rc, ginsenoside $\mathrm{Rc}$; filaggrin, filament aggregating protein.

hydrogen peroxide and hydroxyl radicals. Notably, treatment with Rc was revealed to attenuate the UVB-induced increase in ROS production, thus suggesting that Rc may exert antioxidative effects against UVB-induced photooxidative stress.

MMPs have a key role during collagen degradation and have been implicated in skin photoaging (25). Enhanced ROS levels following UV irradiation have been reported to promote the production and secretion of MMPs, including MMP-2 and MMP-9, in dermal and epidermal tissue, thus leading to skin damage and photoaging (26). In the present study, Rc treatment was revealed to attenuate the UVB-induced upregulation in MMP-2 and MMP-9 protein expression in human keratinocytes. In addition, their proteolytic activity was similarly suppressed. These findings suggested that MMP-2 and MMP-9 protein expression may be regulated by a common mechanism in keratinocytes.

Natural antioxidants have been previously reported to exert their physiological functions through the downregulation of MMP-2 and MMP-9 via various mechanisms (27-29). Photodynamic therapy has been demonstrated to suppress the migration and invasion ability of laryngeal squamous carcinoma cells in vitro, via downregulation of MMP-2 and MMP-9 expression levels through the ROS-mediated inhibition of the mitogen-activated protein kinase (MAPK)/extracellular 
signal-regulated kinase signaling pathway (27). Polysaccharides derived from Inonotus obliquus have been revealed to suppress the migration and invasion ability of highly metastatic melanoma cells in vitro by decreasing the expression and activity levels of MMP-2 and MMP-9 via the inhibition of MAPK, cyclooxygenase-2 and nuclear factor- $\kappa \mathrm{B}$ signaling pathways (28). Amsacrine has been previously reported to downregulate MMP-2 and MMP-9 expression by suppression of gene transcription and promotion of mRNA decay in human leukemia cells (29). The findings of the present study suggested that Rc may simultaneously downregulate the expression of MMP-2 and MMP-9 in keratinocytes under conditions of photooxidative stress; however, additional studies are required to fully elucidate the underlying molecular mechanisms.

The present study revealed that Rc counteracted the UVB-induced depletion of GSH contents and the suppression of SOD activity. These findings suggested that the modulation of endogenous antioxidants may be implicated in the antioxidative properties of Rc; however, additional studies are required to determine the molecular mechanisms that are involved. Numerous antioxidants exert their biological roles through the upregulation of the endogenous antioxidative mechanisms of cells. Fucoxanthin is a natural antioxidant carotenoid, which is abundant in seaweed, and has been previously reported to increase GSH levels by upregulating mRNA and protein expression levels of $\gamma$-glutamylcysteine synthetase and glutathione synthetase in keratinocytes, via promoting the nuclear translocation and phosphorylation of nuclear factor (erythroid-derived 2)-like 2 (30). The essential oil of Pogostemon cablin, which is a Chinese herb traditionally used for the treatment of skin disorders, has been reported to reduce wrinkle formation and increase skin elasticity and collagen content, possibly due to its antioxidative properties by suppressing lipid peroxidation through the potentiation of SOD, glutathione peroxidase and catalase activity (31). Coenzyme Q has also been revealed to prevent UVB-induced photooxidative stress by increasing SOD and glutathione peroxidase activity reduced by UVB radiation in mice (32).

In the present study, exposure to UVB radiation led to the downregulation of filaggrin expression and reduced caspase-14 activity in keratinocytes, thus suggesting the dysfunction of the skin barrier. Rc treatment was revealed to increase filaggrin expression and potentiate caspase-14 activity, thus counteracting the UVB-induced skin barrier impairments. These findings suggested that the antioxidative properties of Rc may be associated with its functions in preserving skin barrier functionality; however, additional studies are required to elucidate the underlying molecular mechanisms.

Previous studies (33-38) have assessed the pharmacological actions of purified ginsenosides, including their anti-photoaging, anti-inflammatory, barrier function-preserving and antioxidative functions, using skin cell lines. Similar to Rc, PPD-type ginsenosides, such as Rb1 (33), Rb2 $(34,35)$ and Rb3 (36), used as enantiomer mixtures, were reported to exhibit anti-photoaging properties in keratinocytes and fibroblasts. The PPD-type ginsenoside Rg3 exhibited a stereoselective anti-photoaging action, as only the $S$-enantiomer was revealed to have a ROS-scavenging and MMP-2 inhibitory effect in keratinocytes (37). In UVB-irradiated keratinocytes, the PPD-type ginsenoside
20(S)-Rh2 reduced ROS generation and MMP-2 expression levels, whereas 20(R)-Rh2 downregulated MMP-2, but did not affect ROS production, thus suggesting that the two enantiomers may exert anti-photoaging effects that involve different molecular mechanisms (38). In addition, 20(R)-Rh2 demonstrated an anti-inflammatory effect in lipopolysaccharide-stimulated macrophages, by downregulating nitric oxide, prostaglandin E2, ROS and MMP-9 levels (39). Notably, 20(S)-, but not 20(R)-PPD, was reported to prevent the UVB-induced upregulation in ROS and MMP-2 levels in keratinocytes, thus suggesting the anti-photoaging potential of 20(S)-PPD-type ginsenosides (40). Furthermore, the PPT-type ginsenoside $\mathrm{Rg} 2$ exhibited stereospecific protective properties, as only the $20(S)$-enantiomer was revealed to prevent UVB-induced photoaging, via upregulation of endogenous antioxidants, such as GSH and SOD (41). Similarly, 20(S)-PPT was previously reported to exert anti-photoaging effects in UVB-irradiated keratinocytes (42). Re, which is a PPT-type ginsenoside, was demonstrated to preserve skin barrier functionality, via enhancing $\mathrm{CE}$ formation, and potentiating filaggrin and caspase-14 activity in skin cells under physiological conditions (43). Ro, an oleanolic acid-type ginsenoside, also exhibited anti-photoaging potential by counteracting the UVB-induced decrease in fibroblast GSH contents (44). These previous findings suggested that ginsenosides may exert beneficial actions on human skin; however, the mechanisms underlying their effects may vary depending on the type of ginsenoside and their stereospecificity.

In conclusion, the present study investigated the putative anti-photoaging and barrier function-preserving properties of Rc in UVB-irradiated human HaCaT keratinocytes. Rc treatment was demonstrated to prevent UVB-induced ROS generation and suppress the expression and activity of MMP-2 and MMP-9 and counteract the UVB-induced GSH depletion and suppression of SOD activity. In addition, Rc was revealed to attenuate the UVB-induced downregulation in filaggrin expression and caspase-14 activity. The findings of the present study suggested that ginsenoside Rc may be a potential target for the development of novel strategies based on natural compounds for skin protection with fewer adverse effects.

\section{Acknowledgements}

The present study was supported by the Ministry of Trade, Industry and Energy, Korea Institute for Advancement of Technology, through the Encouragement Program for the Industries of Economic Cooperation Region (grant no. R0005382).

\section{References}

1. Shi Y, Sun C, Zheng B, Li Y and Wang Y: Simultaneous determination of nineginsenosides in functional foods by high performance liquid chromatography with diode array detector detection. Food Chem 123: 1322-1327, 2010.

2. Harkey MR, Henderson GL, Gershwin ME, Stern JS and Hackman RM: Variability in commercial ginseng products: An analysis of 25 preparations. Am J Clin Nutr 73: 1101-1106, 2001.

3. Kim DH, Park CH, Park D, Choi YJ, Park MH, Chung KW, Kim SR, Lee JS and Chung HY: Ginsenoside Rc modulates Akt/FoxO1 pathways and suppresses oxidative stress. Arch Pharm Res 37: 813-820, 2014. 
4. Lee MS, Hwang JT, Kim SH, Yoon S, Kim MS, Yang HJ and Kwon DY: Ginsenoside Rc, an active component of Panax ginseng, stimulates glucose uptake in $\mathrm{C} 2 \mathrm{C} 12$ myotubes through an AMPK-dependent mechanism. J Ethnopharmacol 127: 771-776, 2010.

5. Peres PS, Terra VA, Guarnier FA, Cecchini R and Cecchini AL: Photoaging and chronological aging profile: Understanding oxidation of the skin. J Photochem Photobiol B 103: 93-97, 2011.

6. Curran S and Murray GI: Matrix metalloproteinases in tumour invasion and metastasis. J Pathol 189: 300-308, 1999.

7. Masaki H, Atsumi T and Sakurai H: Detection of hydrogen peroxide and hydroxyl radicals in murine skin fibroblasts under UVB irradiation. Biochem Biophys Res Commun 206: 474-479, 1995.

8. Kim MS, Oh GH, Kim MJ and Hwang JK: Fucosterol inhibits matrix metalloproteinase expression and promotes type-1 procollagen production in UVB-induced HaCaT cells. Photochem Photobiol 89: 911-918, 2013.

9. Inomata S, Matsunaga Y, Amano S, Takada K, Kobayashi K, Tsunenaga M, Nishiyama T, Kohno Y and Fukuda M: Possible involvement of gelatinases in basement membrane damage and wrinkle formation in chronically ultraviolet B-exposed hairless mouse. J Invest Dermatol 120: 128-134, 2003.

10. McGrath JA and Uitto J: The filaggrin story: Novel insights into skin-barrier function and disease. Trends Mol Med 14: 20-27, 2008

11. Kezic S, O'Regan GM, Lutter R, Jakasa I, Koster ES, Saunders S Caspers P, Kemperman PM, Puppels GJ, Sandilands A, et al: Filaggrin loss-of-function mutations are associated with enhanced expression of IL-1 cytokines in the stratum corneum of patients with atopic dermatitis and in a murine model of filaggrin deficiency. J Allergy Clin Immunol 129: 1031-1039.e1, 2012.

12. Dang NN, Pang SG, Song HY, An LG and Ma XL: Filaggrin silencing by shRNA directly impairs the skin barrier function of normal human epidermal keratinocytes and then induces an immune response. Braz J Med Biol Res 48: 39-45, 2015.

13. Sandilands A, Sutherland C, Irvine AD and McLean WH: Filaggrin in the frontline: Role in skin barrier function and disease. J Cell Sci 122: 1285-1294, 2009.

14. Hvid M, Johansen C, Deleuran B, Kemp K, Deleuran M and Vestergaard C: Regulation of caspase 14 expression in keratinocytes by inflammatory cytokines-a possible link between reduced skin barrier function and inflammation? Exp Dermatol 20: 633-636, 2011.

15. Hibino T, Fujita E, Tsuji Y, Nakanishi J, Iwaki H, Katagiri C and Momoi T: Purification and characterization of active caspase-14 from human epidermis and development of the cleavage site-directed antibody. J Cell Biochem 109: 487-497, 2010.

16. Bradford MM: A rapid and sensitive method for the quantitation of microgram quantities of protein utilizing the principle of protein-dye binding. Anal Biochem 72: 248-254, 1976.

17. Royall JA and Ischiropoulos H: Evaluation of 2',7'-dichlorofluorescin and dihydrorhodamine 123 as fluorescent probes for intracellular $\mathrm{H}_{2} \mathrm{O}_{2}$ in cultured endothelial cells. Arch Biochem Biophys 302: 348-355, 1993.

18. Gomes A, Fernandes E and Lima JL: Fluorescence probes used for detection of reactive oxygen species. J Biochem Biophys Methods 65: 45-80, 2005

19. Freshney RI: Culture of Animal Cells: A Manual of Basic Technique. 4th edition. Wiley-Liss Press, New York, 1994.

20. Kleiner DE and Stetler-Stevenson WG: Quantitative zymography: Detection of picogram quantities of gelatinases. Anal Biochem 218: 325-329, 1994.

21. Nakagawa K, Saijo N, Tsuchida S, Sakai M, Tsunokawa Y, Yokota J, Muramatsu M, Sato K, Terada M and Tew KD: Glutathione-S-transferase pi as a determinant of drug resistance in transfectant cell lines. J Biol Chem 265: 4296-4301, 1990.

22. Lee YY, Kim HG, Jung HI, Shin YH, Hong SM, Park EH, Sa JH and Lim CJ: Activities of antioxidant and redox enzymes in human normal hepatic and hepatoma cell lines. Mol Cells 14: 305-311, 2002

23. Zhu M and Bowden GT: Molecular mechanism(s) for UV-B irradiation-induced glutathione depletion in cultured human keratinocytes. Photochem Photobiol 80: 191-196, 2004.

24. Aitken GR, Henderson JR, Chang SC, McNeil CJ and Birch-Machin MA: Direct monitoring of UV-induced free radical generation in HaCaT keratinocytes. Clin Exp Dermatol 32: 722-727, 2007

25. Fisher GJ, Kang S, Varani J, Bata-Csorgo Z, Wan Y, Datta S and Voorhees JJ: Mechanisms of photoaging and chronological skin aging. Arch Dermatol 138: 1462-1470, 2002 ,
26. Lee YM, Kang SM, Lee SR, Kong KH, Lee JY, Kim EJ and Chung JH: Inhibitory effects of TRPV1 blocker on UV-induced responses in the hairless mice. Arch Dermatol Res 303: 727-736, 2011.

27. Zhang H, Shen B, Swinarska JT, Li W, Xiao K and He P. 9-Hydroxypheophorbide $\alpha$-mediated photodynamic therapy induces matrix metalloproteinase-2 (MMP-2) and MMP-9 down-regulation in Hep-2 cells via ROS-mediated suppression of the ERK pathway. Photodiagnosis Photodyn Ther 11: 55-62, 2014.

28. Lee KR, Lee JS, Kim YR, Song IG and Hong EK: Polysaccharide from Inonotus obliquus inhibits migration and invasion in B16-F10 cells by suppressing MMP-2 and MMP-9 via downregulation of NF-אB signaling pathway. Oncol Rep 31: 2447-2453, 2014.

29. Liu WH, Chen YJ, Chien JH and Chang LS: Amsacrine suppresses matrix metalloproteinase-2 (MMP-2)/MMP-9 expression in human leukemia cells. J Cell Physiol 229: 588-598, 2014.

30. Zheng W, Zhang Y, Ma D, Shi Y, Liu C and Wang P: ( \pm Equol inhibits invasion in prostate cancer DU145 cells possibly via down-regulation of matrix metalloproteinase-9, matrix metalloproteinase-2 and urokinase-type plasminogen activator by antioxidant activity. J Clin Biochem Nutr 51: 61-67, 2012.

31. Lin RF, Feng XX, Li CW, Zhang XJ, Yu XT, Zhou JY, Zhang X, Xie YL, Su ZR and Zhan JY: Prevention of UV radiation-induced cutaneous photoaging in mice by topical administration of patchouli oil. J Ethnopharmacol 154: 408-418, 2014.

32. Kim DW, Hwang IK, Kim DW, Yoo KY, Won CK, Moon WK and Won MH: Coenzyme Q_ $\{10\}$ effects on manganese superoxide dismutase and glutathione peroxidase in the hairless mouse skin induced by ultraviolet B irradiation. Biofactors 30: 139-147, 2007.

33. Oh SJ, Kim K and Lim CJ: Protective properties of ginsenoside $\mathrm{Rb} 1$ against UV-B radiation-induced oxidative stress in human dermal keratinocytes. Pharmazie 70: 381-387, 2015.

34. Oh SJ, Kim K and Lim CJ: Ginsenoside Rb2 attenuates UV-B radiation-induced reactive oxygen species and matrix metalloproteinase-2 through upregulation of antioxidant components in human dermal fibroblasts. Pharmacology 96: 32-40, 2015.

35. Oh SJ, Kim K and Lim CJ: Suppressive properties of ginsenoside $\mathrm{Rb} 2$, a protopanaxadiol-type ginseng saponin, on reactive oxygen species and matrix metalloproteinase-2 in UV-B-irradiated human dermal keratinocytes. Biosci Biotechnol Biochem 79: 1075-1081, 2015.

36. Oh SJ, Oh Y, Ryu IW, Kim K and Lim CJ: Protective properties of ginsenoside $\mathrm{Rb} 3$ against UV-B radiation-induced oxidative stress in HaCaT keratinocytes. Biosci Biotechnol Biochem 80: 95-103, 2015.

37. Lim CJ, Choi WY and Jung HJ: Stereoselective skin anti-photoaging properties of ginsenoside $\mathrm{Rg} 3$ in UV-B-irradiated keratinocytes. Biol Pharm Bull 37: 1583-1590, 2014

38. Oh SJ, Lee S, Choi WY and Lim CJ: Skin anti-photoaging properties of ginsenoside Rh2 epimers in UV-B-irradiated human keratinocyte cells. J Biosci 39: 673-682, 2014.

39. Choi WY, Lim HW and Lim CJ: Anti-inflammatory, antioxidative and matrix metalloproteinase inhibitory properties of 20(R)-ginsenoside Rh2 in cultured macrophages and keratinocytes. J Pharm Pharmacol 65: 310-316, 2013.

40. Oh SJ, Lee S, Kho YE, Kim K, Jin CD and Lim CJ: Stereoselective suppressive effects of protopanaxadiol epimers on UV-B-induced reactive oxygen species and matrix metalloproteinase- 2 in human dermal keratinocytes. Can J Physiol Pharmacol 93: 91-95, 2015.

41. Kang HJ, Huang YH, Lim HW, Shin D, Jang K, Lee Y, Kim K and Lim CJ: Stereospecificity of ginsenoside Rg2 epimers in the protective response against UV-B radiation-induced oxidative stress in human epidermal keratinocytes. J Photochem Photobiol B 165: 232-239, 2016

42. Oh SJ, Kim K and Lim CJ: Photoprotective properties of 20(S)-protopanaxatriol, an aglycone of ginseng saponins: Protection from ultraviolet-B radiation-induced oxidative stress in human epidermal keratinocytes. Mol Med Rep 14: 2839-2845, 2016.

43. Oh Y, Lim HW, Kim K and Lim CJ: Ginsenoside Re improves skin barrier function in $\mathrm{HaCaT}$ keratinocytes under normal growth conditions. Biosci Biotechnol Biochem 13: 1-3, 2016.

44. Kang HJ, Oh Y, Lee S, Ryu IW, Kim K and Lim CJ: Antioxidative properties of ginsenoside Ro against UV-B-induced oxidative stress in human dermal fibroblasts. Biosci Biotechnol Biochem 79: 2018-2021, 2015. 